\title{
Research on the Method of Traffic Organization and Optimization Based on Dynamic Traffic Flow Model
}

\author{
Shu-bin Li, ${ }^{1}$ Guang-min Wang, ${ }^{2}$ Tao Wang, ${ }^{3}$ and Hua-ling Ren ${ }^{4}$ \\ ${ }^{1}$ Department of Traffic Management Engineering, Shandong Police College, Jinan 250014, China \\ ${ }^{2}$ School of Economics and Management, China University of Geosciences, Wuhan 430074, China \\ ${ }^{3}$ School of Automation and Electronic Engineering, Qingdao University of Science and Technology, Qingdao 266042, China \\ ${ }^{4}$ School of Traffic and Transportation, Beijing Jiaotong University, Beijing 100044, China \\ Correspondence should be addressed to Shu-bin Li; li_shu_bin@163.com
}

Received 23 November 2016; Revised 7 February 2017; Accepted 16 February 2017; Published 14 March 2017

Academic Editor: J. R. Torregrosa

Copyright (c) 2017 Shu-bin Li et al. This is an open access article distributed under the Creative Commons Attribution License, which permits unrestricted use, distribution, and reproduction in any medium, provided the original work is properly cited.

\begin{abstract}
The modern transportation system is becoming sluggish by traffic jams, so much so that it can harm the economic and society in our country. One of the reasons is the surging vehicles day by day. Another reason is the shortage of the traffic supply seriously. But the most important reason is that the traffic organization and optimization hardly met the conditions of modern transport development. In this paper, the practical method of the traffic organization and optimization used in regional area is explored by the dynamic traffic network analysis method. Firstly, the operational states of the regional traffic network are obtained by simulation method based on the self-developed traffic simulation software DynaCHINA, in which the improved traffic flow simulation model was proposed in order to be more suitable for actual domestic urban transport situation. Then the appropriated optimization model and algorithm were proposed according to different optimized content and organization goals, and the traffic simulation processes more suitable to regional optimization were designed exactly. Finally, a regional network in Taian city was selected as an example. The simulation results show that the proposed method is effective and feasible. It can provide strong scientific and technological support for the traffic management department.
\end{abstract}

\section{Introduction}

In recent years, ITS (Intelligent Transportation System) has developed rapidly since 2011 and will enter into the mature period in China after 2020. But with the increased of vehicles and road construction, the latter is far behind the former, so a series of unavoidable traffic problems were triggered such as traffic congestion, traffic safety, and environment pollution. In order to resolve the contradiction of traffic supply and demand, a viable method is the national policy and the other is to develop intelligent transportation technologies. The latter can optimize transportation resources from all aspects of traffic supply. The traffic organization and optimization are a better way to optimize the existing transportation facilities by using the method of system engineering under the guidance of system science. It can reasonably adjust the relationship between traffic demand and traffic supply so as to make better use of road resources. Moreover the road can play an important part in traffic system by scientific organization and distribution. Further the traffic flow can be smooth and safety. The traffic congestion can be reduced to a certain degree [1].

The content of traffic organization and optimization varies with different situations. Shi et al. [2] raised the optimization approach of one-way traffic organization in urban micro circulation transportation network by the constraint of road saturation. Long et al. [3] proposed the different model and algorithm for the same problem. Dussutour et al. [4] studied the optimization behavior of ants, whose contribution is published in "Nature." Jia [5] studied the problem of traffic organization optimization on urban main road systematically and realized the two-direction green pass. Xu et al. [6] carried out the traffic organization optimization by using simulation method for road traffic bottlenecks; 
its purpose is to reduce traffic congestion. Yao et al. [7] proposed a more practical method of information induced and simulation; in a large flow road the micro circulation method was put forward. Yang et al. [8, 9] proposed the regional traffic organization methods integrated time period; their suggestions and measures were effective. Sun et al. [10, 11] proposed also some valuable suggestions for the urban passenger transport hub by simulation method. Zhengyan [12] addressed some technical method for traffic organization of emergency evacuation.

From the above document analysis, most papers focus on the traffic flow for traffic organization and optimization method. In order to ensure reasonableness and effectiveness of traffic organization, the pros and cons are needed to be considered. In this paper, the dynamic method is proposed in view of the shortcomings of the static method, such as the peak period and flat peak period of traffic flow ware considered.

To establish a scientific and effective traffic management and optimization scheme, it is necessary to analyze relationship of the traffic demand and traffic supply. By forecasting interaction of the traffic demand and traffic supply realtime, the response behavior of the drivers can be simulated, and then the traffic control scheme and the traffic guidance strategy were analyzed. The self-developed software system DynaCHINA was used to do some work in this spot.

\section{Overview of DynaCHINA}

This paper presents a new simulation-based DTA (dynamic traffic assignment) system: DynaCHINA (Dynamic Consistent Hybrid Information Based on Network Assignment). It is the only self-developed software system in China by our team. Although the basic framework of DynaMIT is adopted in its implementation, compared with DynaMIT and DynaSMART, DynaCHINA has four unique features: modeling mixed traffic and special driver behavior; availability of floating car data (FCD) in urban networks; anisotropic mesoscopic supply simulator; availability of true OD flows in freeway networks. Predictive capacity is the core of DynaCHINA. DynaCHINA shows the potential for realtime traffic prediction application according to the laboratory evaluation results with data collected from real activity. It is the first and the only Traffic Estimation and Prediction System (TrEPS) in China.

DynaCHINA integrates the advanced dynamic traffic demand analysis technology, the dynamic traffic assignment technology, many discrete choice models, off-line and online system parameters calibration method, and so forth. The software can provide simulation test, analysis, and evaluation method for the related research and implementation of intelligent transportation system. It has the ability to estimate and forecast the network traffic flow, density, and speed which will provide technical support for the traffic control and traffic guidance system. It makes these systems more effective to ease traffic congestion and improve the efficiency of travel.

DynaCHINA contains many mathematical models, such as dynamic OD estimation model, mesoscopic traffic simulation model, vehicle moving model, speed-density model, queuing model, and network model. At the same time, in order to achieve the purpose of online application, the efficient algorithms were designed, such as path generation algorithm and simulation steps of the specific design. The developed prototype is basically completed; the source code reached tens of thousands. Some other functions of bus simulation and VIP route guidance for specially vehicles were also proposed.

DynaCHINA targets more realistic approaches to shortterm transportation planning such as special events and work zones and addresses the growing importance of real-time applications such as incident management, route guidance, and emergency response. It is capable of providing predictive traffic information (speeds, densities, and queues/flows) based on a rolling-horizon implementation of an assignmentsimulation framework. DynaCHINA can be operated in a distributed computation mode in support of real-time operations, including data synchronization, self-calibration of system parameters, and self-correcting of system states. Currently DynaCHINA team is working with some agencies to conduct laboratory experiment and initial field deployments of the prototypes to investigate functionality, prediction accuracy, computational efficiency, and robustness in the field environment at Beijing, Jinan, and Guangzhou. Figure 1 shows how DynaCHINA can be implemented.

\section{The Mesoscopic Traffic Simulation Models and Algorithm}

There are a lot of modes developed in DynaCHINA such as road network model, the traveler behavior model, the path generation algorithm, and the capacity models. In these models, the mesoscopic traffic simulation model and the dynamic OD estimation model are the most important ones. The proposed simulation process is designed efficiently. The following focuses on the models and algorithm.

3.1. The Mesoscopic Traffic Simulation Models. There are two key models in the mesoscopic simulation process. One is the speed-density relationship model. It needs some information of road flow, speed, or density in order to more accurately reproduce the current road traffic condition. In general, one or more parameters must be calibrated in the following function:

$$
v_{u}=\max \left\{v_{\min }, v_{\max }\left\{1-\left\{\frac{k-k_{\max }}{k_{\mathrm{jam}}}\right\}^{\beta}\right\}^{\alpha}\right\},
$$

where $v_{u}$ is the vehicle speed, $k$ is the density, $k_{\text {jam }}$ is the jam density, $k_{\max }$ is the critical density, $v_{\min }$ and $v_{\max }$ are the minimum and maximum speed designed. In this paper, the traffic simulation technology is used more than one time; the minimum speed is designed to avoid the situation of being unable start the simulation system. In order to be more realistic, the problem can be described as that the upstream of a long road has a constant speed and followed the 


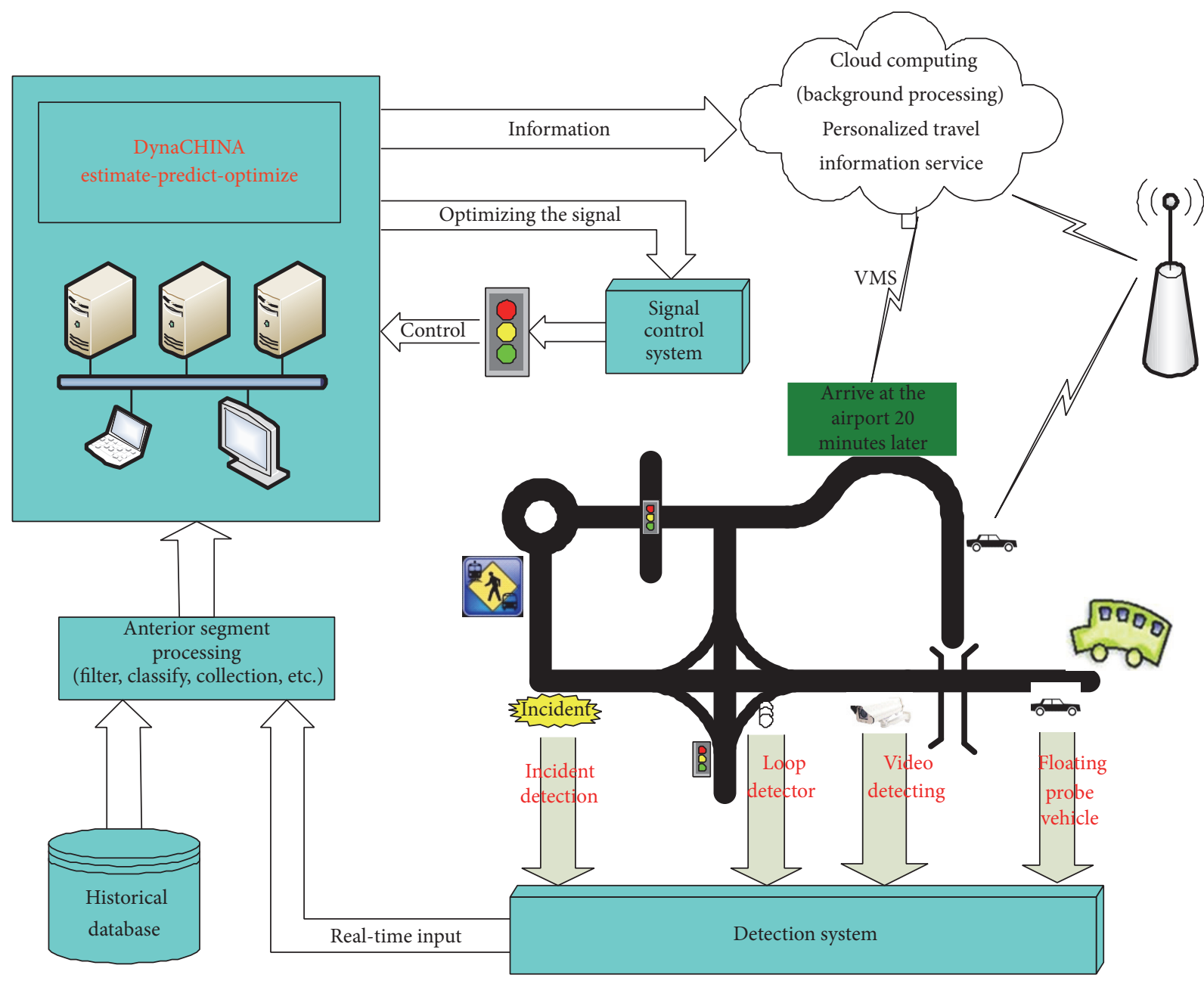

Figure 1: DynaCHINA system principle.

speed-density relationship at the downstream. Mathematical description is as follows:

$$
\begin{aligned}
& v_{u} \\
& = \begin{cases}v_{f} & k \leq k_{\max } \\
\max \left\{v_{\min }, v_{\max }\left\{1-\left\{\frac{k-k_{\max }}{k_{\mathrm{jam}}}\right\}^{\beta}\right\}\right. & k>k_{\max },\end{cases}
\end{aligned}
$$

where $\alpha$ and $\beta$ are parameters.

The other is the vehicle movement model. Its function is mainly to put the vehicles at the right position based on the speed of vehicles on the network. This also means the relationship between time and position.

$$
t(z)= \begin{cases}\frac{1}{\lambda} \log \frac{\lambda_{z+v_{u}}}{\lambda_{z_{0}+v_{u}}} & \text { if } v_{u} \neq v_{d} \\ \frac{z-z_{0}}{v_{u}} & \text { if } v_{u}=v_{d}\end{cases}
$$

where $t$ is the time and $z$ is the position; when $t=0$, the position is $z_{0} . v_{d}$ is the downstream vehicle speed; usually parameter $\lambda$ is consistent with the following formula:

$$
\lambda=\frac{v_{d}-v_{u}}{L_{s}}
$$

$L_{s}$ is the length of the deceleration/acceleration zone of the road.

3.2. The Estimation Method of Dynamic OD Demand. In simulation period, how many vehicles can be loaded on the network? The real-time OD estimation model must be needed. In this section, the generalized least square estimation model is used to resolve the problem.

$$
\widehat{\mathbf{x}}_{h}=\arg \min \left[\left(\mathbf{x}_{h}-\mathbf{x}_{h}^{a}\right)^{T} \mathbf{W}_{h}^{-1}\left(\mathbf{x}_{h}-\mathbf{x}_{h}^{a}\right)\right.
$$




$$
\begin{aligned}
& +\left(\mathbf{y}_{h}-\sum_{p=h-p^{\prime}}^{h-1} \mathbf{a}_{h}^{p_{\mathbf{x}_{p}}}-\mathbf{a}_{h}^{h} \mathbf{x}_{h}\right)^{T} \\
& \left.\cdot \mathbf{R}_{h}^{-1}\left(\mathbf{y}_{h}-\sum_{p=h-p^{\prime}}^{h-1} \mathbf{a}_{h}^{p} \widehat{\mathbf{x}}_{p}-\mathbf{a}_{h}^{h} \mathbf{x}_{h}\right)\right],
\end{aligned}
$$

where $\widehat{\mathbf{x}}_{h}$ is the estimated OD matrix; $\mathbf{x}_{h}^{a}$ is the historical OD matrix which refers to a priori values of $\mathbf{x}_{h}$ in the time period $h ; n_{l}$ is the section number with monitoring equipment; $\mathbf{y}_{h} \in$ $R^{n_{l}}$ is the monitored value on section $n_{l}$ in time period $h ; \mathbf{a}_{h}^{p}$ $(p=h, h-1, \ldots, h-p)$ is the dynamic traffic assignment matrix which defined a mapping between the OD vector $\mathbf{x}_{p}$ in $p$ and the traffic volume vector in $h$; if there is not any a priori information, $\mathbf{W}_{h}$ and $\mathbf{R}_{h}$ can be defined as a unit matrix [13].

Another dynamic OD demand estimation model is Kalman filtering model. It includes state and space model. The basic idea is proposed by Ashok and Ben-Akiva [14] in 1933. Unlike other approaches in the literatures, the approach is based on deviations from historical values. Considering a network with $n_{\mathrm{OD}}$ OD pairs and $n_{l}$ link counts, let us assume the following notation:

$\mathbf{x}_{h+1}$ is the matrix representing the number of vehicles between each OD pair departing their origins during time interval $h$ and $x_{h}^{H}$ is the corresponding historical estimated matrix. The transition equation can be expressed as

$$
\boldsymbol{\delta} \mathbf{x}_{h+1}=\sum_{p=h+1-q^{\prime}}^{h} \mathbf{f}_{h+1}^{p} \boldsymbol{\delta} \mathbf{x}_{p}+\mathbf{w}_{h+1}
$$

where $\mathbf{f}_{h}^{p}$ is a $n_{\mathrm{OD}} \times n_{\mathrm{OD}}$ matrix mapping from $\left(x_{p}-x_{p}^{H}\right)$ to $\left(x_{h+1}-x_{h+1}^{H}\right), \mathbf{w}_{h+1}$ is a $\left(n_{\mathrm{OD}} \times 1\right)$ vector of random errors, and $q^{\prime}$ is the degree of the autoregressive process.

The error is assumed as follows:

$$
\begin{aligned}
E\left(\mathbf{w}_{h+1}\right) & =0, \\
E\left(\mathbf{w}_{h} \mathbf{w}_{l}^{T}\right) & =\mathbf{Q}_{h} \delta_{h l},
\end{aligned}
$$$$
\mathbf{Q}_{h} \text { is covariance matrix, } \delta_{h l}= \begin{cases}1, & h=l \\ 0, & h \neq l .\end{cases}
$$

The measurement equation which relates unknown OD flows to the observed link counts can be stated mathematically as follows:

$$
\boldsymbol{\delta} \mathbf{y}_{h}=\sum_{p=h-p^{\prime}}^{h} \mathbf{a}_{h}^{p} \boldsymbol{\delta} \mathbf{x}_{p}+\mathbf{v}_{h}
$$

where $\mathbf{a}_{h}^{p}$ is an assignment matrix of contributions from $\mathbf{x}_{p}$ to $\mathbf{y}_{h} ; \boldsymbol{\delta} \mathbf{y}_{h}=\mathbf{y}_{h}-\mathbf{y}_{h}^{H}=\mathbf{y}_{h}-\sum_{p=h-p^{\prime}}^{h} \mathbf{a}_{h}^{p} \mathbf{x}_{p}^{H}, \mathbf{y}_{h}^{H}$ are the link flows obtained by assigning the historical OD flows, $\mathbf{y}_{h}$ is the detection value of the traffic volume of the road section in the $h$ time, $p^{\prime}$ is the number of time intervals corresponding to the longest trip; $\mathbf{v}_{h}$ is the Gauss white noise which is independent of the system noise and satisfies $E\left(v_{h}\right)=0$, and $E\left(v_{h} v_{l}^{T}\right)=\mathbf{R}_{h} \delta_{h l}$. Equations (6) and (8) constitute the state and space model of the dynamic OD matrix estimation.

3.3. Simulation Process. The designed simulation process is very important to the simulation accuracy. The simulation time is divided into a number of equal lengths of Update phase. One Update phase is divided into several equal lengths of the Advance interval. In Advance phases, the vehicles can be moved to their new positions. The Update phase is used for updating the traffic dynamics parameters (e.g., densities, speeds, and flow) used in the simulation during each simulation cycle. If the simulation horizon is $T$, without loss of generality, we assume that the time horizon starts at time $t=0$ and finishes at time $t=T$. Update phase is $\Delta t_{\text {update; }}$; Advance phase is $\Delta t_{\text {advance. }}$. So,

$$
\begin{aligned}
T & =k_{u} \Delta t_{\text {update }} \\
\Delta t_{\text {update }} & =k_{A} \Delta t_{\text {advance }}
\end{aligned}
$$

$k_{u}$ and $k_{A}$ are the positive integers; the specific simulation process is as follows.

Step 1 (initialization, input the following information). The network description, the simulation horizon, and the description of all vehicles at the beginning of the interval include their position on the network, their destination, and their current path. All vehicles present in the network at time $t=0$ and the loaded positions [15].

Step 2 (sort the nodes of the traffic network). Set up the network node, section, and other parameters; execute the following program.

Step 2.1. Every segment definition is updated according to the incident description. The output capacities of all segments are updated. Initialize the output counter of the output capacity and initialize the input counter of the acceptance capacity.

Step 2.2. For each segment, compute the upstream-speed and the density. For each direction of the segment, compute the downstream-speed.

Step 2.3. Calculate the speed of the downstream segment, and update the acceptance and output capability of the next segment.

Step 2.4. For a vehicle execute Advance phase; call formulas (9) and (10); load the updated vehicles to their new position of the network.

Step 2.5. $i=0$.

Step 3 (stopping criteria). The algorithm is stopped when all Advance phase intervals have been processed; that is, $i=k_{A}$.

The detailed process is illustrated in Figure 2. 


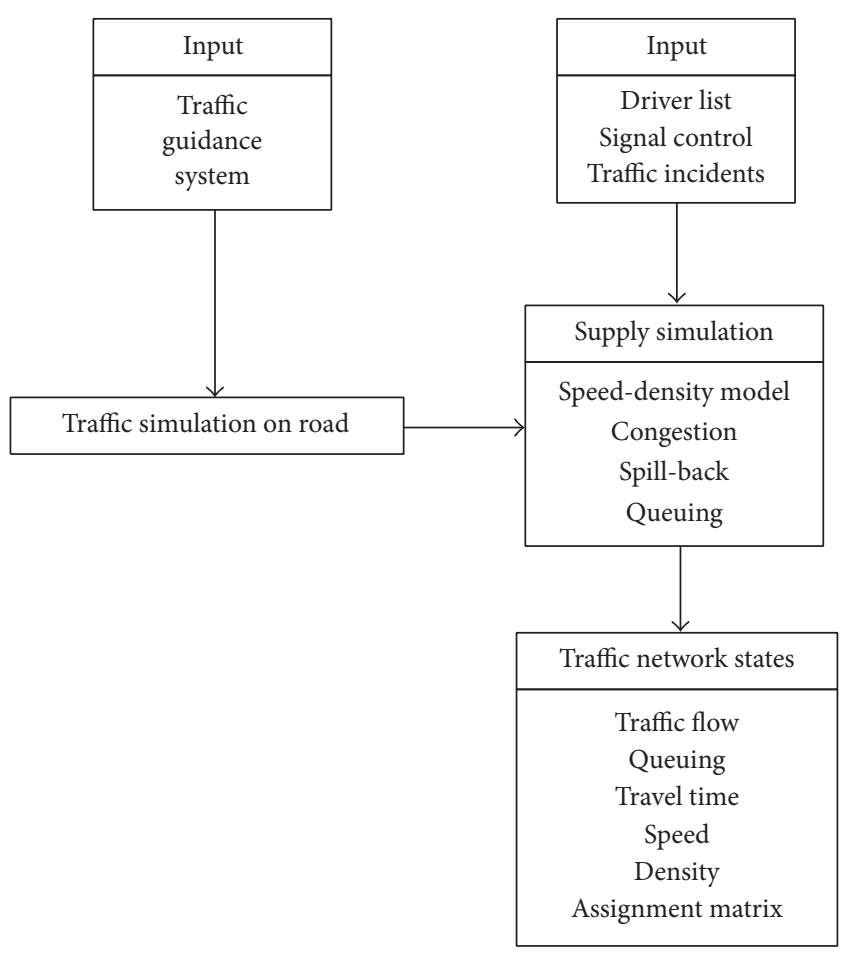

FIGURE 2: General process of simulation.

\section{The Optimization Model and Algorithm of Traffic Organization}

The static traffic organization mainly refers to traffic signs, traffic markings, boot device and parking management, and so forth. There are all the static characteristic. That means it will not change in a long time once the organization method is determined. In contrast, the dynamic method is more flexible and will be changed with time under different traffic network conditions, such as signal control and information. But in any kind of traffic organization, the purpose is to alleviate the traffic pressure of the road network. It comes to it by balancing traffic flow on the network in time and space.

In this paper, the traffic optimization and organization are carried out by using the combination of the static channelized intersection and the signal optimization. Firstly, the division of the road is implemented on intersection level by the way of road channelization. And then, the signal control scheme is set up by using the method of regional signal optimization and by means of simulation to realize it.

The proposed signal optimization model is as follows:

$$
\begin{aligned}
& \min L(\cdot) \\
& =\frac{1}{2}(\mathbf{D}(h))^{2}+\frac{1}{2} \sum_{h=1}(\mathbf{D}(h))^{2}-\sum_{h}(q+f) \\
& \quad+\sum_{h} s .
\end{aligned}
$$

Constraint conditions is as follows:

$$
\begin{aligned}
T_{\min } & \leq T_{h} \leq T_{\max }, \\
g_{\min } & \leq g_{h} \leq g_{\max }, \\
C & =\sum g, \\
C_{\min } & \leq C \leq C_{\max } .
\end{aligned}
$$

$C$ is the total cycle length; $C_{\max }, C_{\min }$ are the maximum and minimum cycle length;

$d=\left\{d_{h}\right\}: d_{h}$ is the column vectors for all vehicle delays in the $h$ time period.

$g$ is the effective green time; $g_{\max }, g_{\min }$ are the maximum and minimum effective green time.

$q=\left\{q_{h}\right\}$ is the total queue length of the network in simulation period; $q_{h}$ is the average queue length of the $h$ period of time all sections of the column vector.

$f=\left\{f_{h}\right\}$ is the sum of all intersection crossing traffic in simulation period; $f_{h}$ is the flow vector of all the inlet channels of $h$ time period.

$T=\left\{T_{h}\right\}$ : timing parameters of all signal controllers in the network.

$T_{h}=\left\{t_{i h}\right\}$ : timing parameter vector of all signal intersections in $h$ time period.

$t_{a h}=\left[t_{a 0 h}^{g}, t_{a 1 h}^{g}, \ldots, t_{a h}^{g}\right]^{T}$ or $\left[C_{\kappa} s_{a 0 h}^{g}, C_{\kappa} s_{a 1 h}^{g}, \ldots\right.$, $\left.C_{\kappa} s_{a 0 h}^{g}\right]^{T}$ is the green time of each phase of the $a$ intersection in $h$ or phase difference of initial phase, $C_{\kappa}$ is the phase number of a signal intersection, and $s_{a b h}^{g}$ is the green-time-rate in $h$, the $a$ intersection, and the $b$ phase.

$T_{\max }, T_{\min }$ : the upper and lower bound constraint vector of signal timing parameters.

$L(\cdot)$ is the objective function.

The SPSA (Simultaneous Perturbation Stochastic Approximation) algorithm is used to solve the problem and also implemented in the simulation system [16]. The SPSA approximates the gradient of the objective function through finite differences. Critically, the approach infers the components of the gradient vector from two function evaluations, after perturbing all components of the parameter vector simultaneously. The computational savings are thus significant when compared to traditional stochastic approximation methods, though many replications may be required in order to obtain a more stable gradient through smoothing.

The SPSA algorithm requires calculation only twice for the objective function independent of the number of parameters $n$. Generally speaking, the SPSA algorithm will produce a series of parameter estimators; that is, the gradient of objective function gradually tends to 0 . Among them, the $i$ iteration of the parameters is updated by

$$
\theta^{i+1}=\theta^{i}-a_{i} \widehat{g}\left(\theta^{i}\right)
$$


where $\theta^{i}$ is parameter vector when the $i$ iteration starts, $\widehat{g}\left(\theta^{i}\right)$ is the current estimator of the vector, and $a_{i}$ is gain for the step length.

The SPSA algorithm is only through two evaluations of the function, the gradient approximation can be got, and the calculation formula is as follows:

$$
\widehat{g}\left(\theta^{i}\right)=\frac{z\left(\theta^{i}+c^{i} \Delta_{i}\right)-z\left(\theta^{i}-c^{i} \Delta_{i}\right)}{2 c^{i}}\left[\begin{array}{c}
\Delta_{i 1}^{-1} \\
\Delta_{i 2}^{-1} \\
\vdots \\
\Delta_{i k}^{-1}
\end{array}\right],
$$

where $\Delta_{i}$ is a $K$-dimensional stochastic perturbation vector; as the numerator for all $k=1,2, \ldots, K$ are the same, so each iteration has the static calculation (nothing to do with the vector dimension $K$ ), which is the most prominent advantages of the algorithm. Of course, the algorithm is required to achieve convergence that must have enough rational number of iterations and then have a good application value. The SPSA algorithm is described below in detail by the steps:

(1) Initialization and coefficient selection: set counter index: $k=0, i=0$, and $\theta^{i}=\theta^{0}$ are initial value of $K$-dimensional optimization vector and pick initial guess nonnegative coefficients $a, A, c, \alpha$, and $\gamma$ in the SPSA.

(2) Set iteration number of calculated gradient vectors in each step: grad_rep; that is, in each iteration step, by many times, take the average gradient estimator.

(3) The iteration counter plus 1; calculate the step length: $a i=a /(A+i) \alpha ; c i=c / i$.

(4) Generate a $K$-dimensional perturbation stochastic vector of independent $\Delta_{i}$; each of its elements $\Delta_{i k}$, $k=1,2, \ldots, K$ is received by a probability distribution. The probability distribution density function is symmetry by the vertical axis (0 axis); $\left|\Delta_{i k}\right|$ and $E\left|\Delta_{i k}^{-1}\right|$ have upper bound. Reference [16] showed that Bernoulli distribution has these characteristics, and uniform distribution and normal conditions are not met.

(5) Make use of the mesoscopic traffic flow model or other traffic simulation systems accessed the values of the objective function by $\theta^{i+}=\theta^{i}+c^{i} \Delta_{i}$ and $\theta^{i-}=\theta^{i}-c^{i} \Delta_{i}$, and each point is required to meet the upper and lower limits constraints in the optimization problem.

(6) According to (9) calculate the $K$-dimensional gradient vector of the stochastic approximation, in which one component of the gradient vector of the $K$ has the same elements, which make the SPSA algorithm different from the traditional finite difference method (FD).

(7) Repeat (4)-(6) steps grad_rep times; each time has an independent sample of $\bar{\Delta}_{i}$, and then finally acquire average gradient vector at $\theta i$.

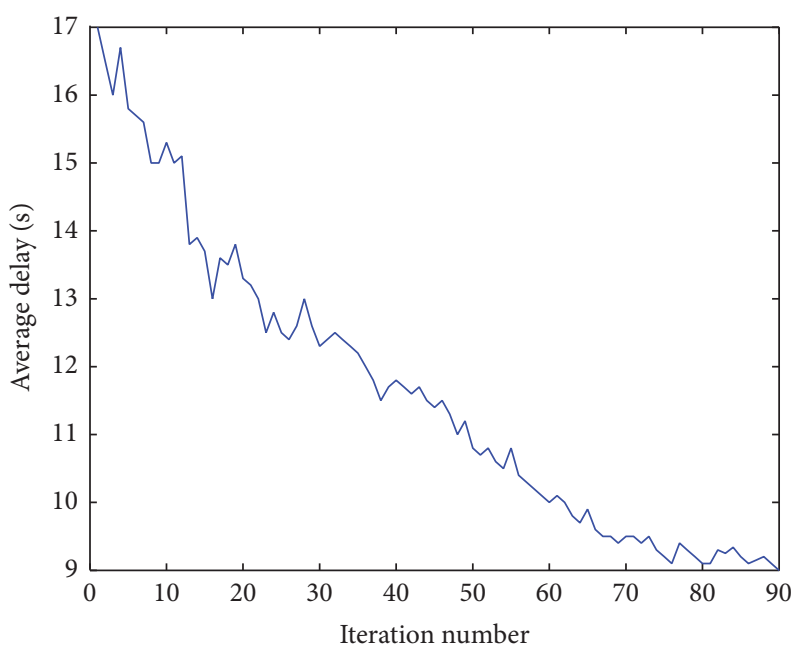

FIGURE 3: Convergence performance of SPSA algorithm.

(8) A new solution point $\theta^{i+1}$ is got from (8), and in accordance with the parameter vector of the upper and lower bound adjust the solution point.

(9) Go back to step (3), continue to iterate until convergence. When the number of iterations to reach the maximum allowable number of iterations or function value $z\left(\theta^{i}\right)$ corresponding to $\theta^{i}$ is little change in several successive iterates, terminate the algorithm.

The SPSA method outlined in this paper immediately illustrates the potential computational savings for large-scale optimization. Figure 3 is the convergence performance of the SPSA algorithm.

\section{Case Study}

In this paper, the real regional network of Tai'an city is used to the case study. Daizong street of Tai'an city in Shandong province in China located in the north of the city and next to famous mountain of Tai. It is about 1.68 kilometers long with 7 signal intersections. The major intersections have a large traffic pressure in rush hour, especially in the morning rush from 7:30 am to 8:30 am. In another time, the traffic flow is the relatively smaller one. In peak hour, the vehicles speed is about $10 \sim 15 \mathrm{~km} / \mathrm{h}$. By survey, the main problem is the badly traffic optimization and organization. There are not reasonable. The capacity of the street decreased sharply by the multitraffic flow from different directions. The marking is not so ideal also. The traffic signal lacks rationality settings which cause serious traffic congestion.

After an investigation in 2016, the hour traffic flow of some day is shown in Figure 4.

Based on the mentioned reasons, the idea of traffic organization in this area is as follows:

(1) Rechanneling intersection: set traffic signs and marking, vehicles organization, and nonmotor vehicles and pedestrian traffic organization.

(2) Calculating signal timings of intersections: set the signal timings according to the traffic flow of the 


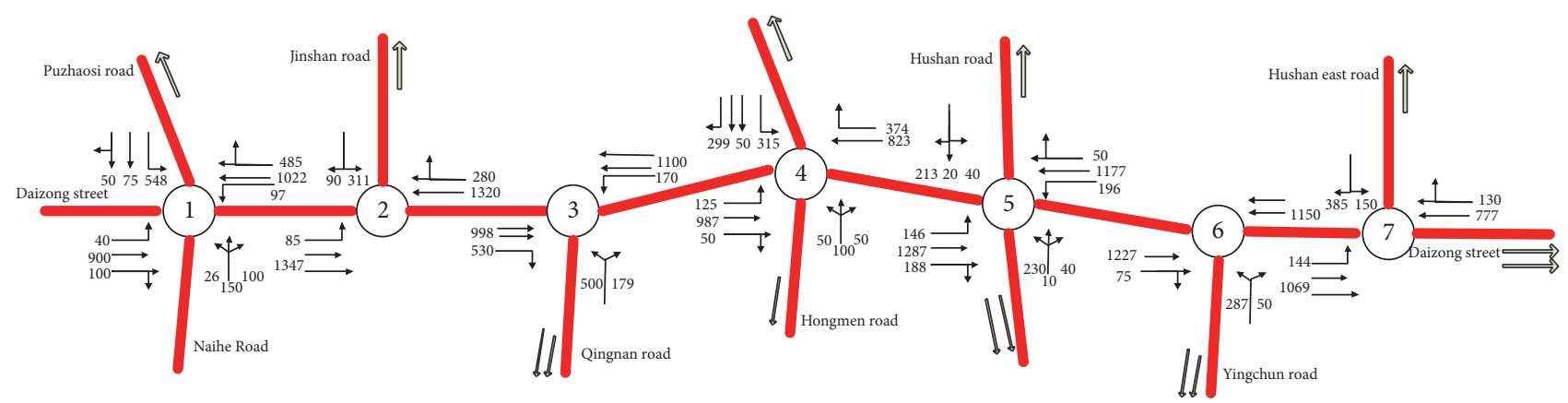

FIgURE 4: Traffic flow of Daizong street.

intersection. In peak hour, execute green pass scheme on main road of the street in order to maximize the through flow. At the same time, control the input traffic flow of intersections on the street.

(3) Executing the proposed mothed of signal optimization in this paper.

Figure 5 is the comparison of the distribution of traffic flow and the detected flow by the parameter calibration method in this paper. The calculation method of the error estimation of RMSN is as follows:

$$
\mathrm{RMSN}=\sqrt{\frac{\sum_{h} \sum_{i}\left(y_{h i}-\widehat{y}_{h i}\right)^{2}}{\sum_{h} \sum_{i} \hat{y}_{h i}^{2}}},
$$

where $y_{h i}$ and $\hat{y}_{h i}$ are the actual measured value and the estimated ones in the $h$ period by the $i$ detector, respectively. As can be seen from Figure 5, the proposed method can estimate the traffic condition of the network accurately. At this point, the simulated road network is equivalent to the actual road network, so the simulation results of the signal control parameters can be as effective as the actual road network.

Figure 3 is the performance index (PI) of iterative optimization of SPSA curve; PI is the average delay by all vehicles on the network. The SPSA algorithm optimized signal timing scheme compared to the Synchro software-based one; the average travel delay of vehicles decreased by $46.67 \%$.

The surveyed data were input into the simulation system. The results show that the traffic flow estimation error was less than $10 \%$ which means the system was reliable. The averaged traffic flow, density, and speed of segments can be contrasted by the traffic organization method. Moreover the designed simulation system can be executed online for implementing the dynamic traffic organization scheme.

The simulation time was two hours. The simulation interval was fifteen minutes, so there were eight intervals. The loaded data was cycled double times survey data. The parameters of the traffic simulation model were the same as [16].

If there were not any the traffic organization optimization measures, the averaged density, speed, and flow can be obtained; see Figures 6-8. From the whole simulation time, the maximum averaged density of each segment is 0.2442

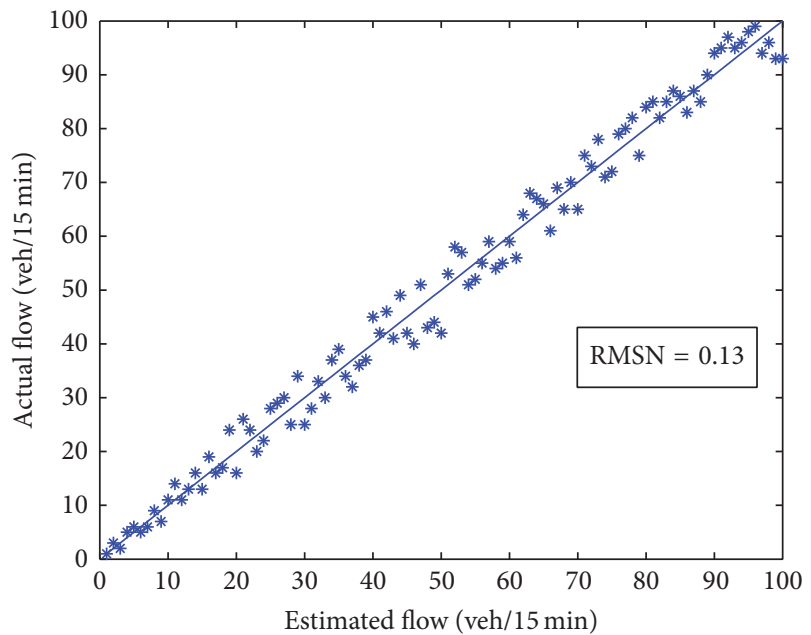

FIGURE 5: Comparison between surveillance flows and estimation flows.

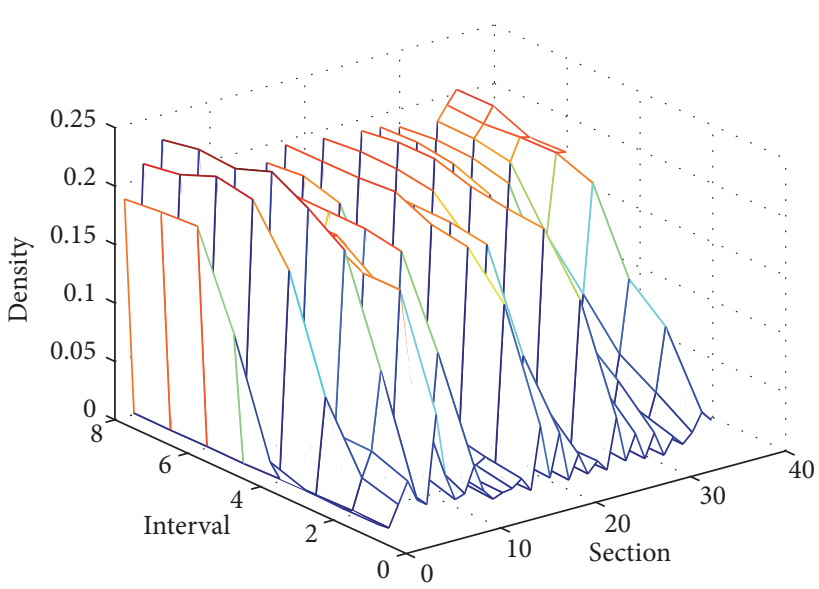

FIGURE 6: Averaged density before optimization.

which existed on the fifth segment in the fifth interval. It was the most crowded at this time. The averaged density and sum density of the network in the whole simulation time were 0.0777 and 22.3873, respectively. Use the same method to analysis the averaged flow and speed. The total flow was 38915. The averaged flow of the network was 135.1215. The 


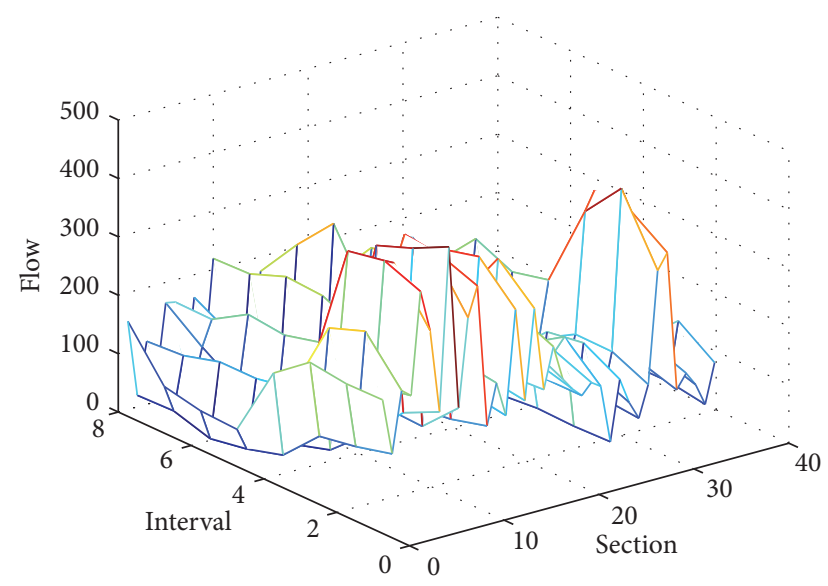

FIgURE 7: Averaged flow before optimization.

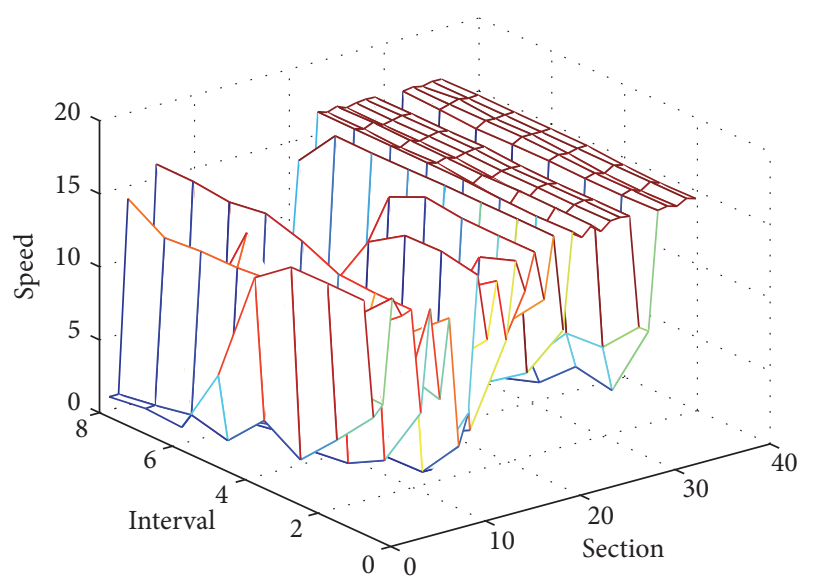

FIGURE 8: Averaged speed before optimization.

maximum flow was 448 on the eighth segment in the first interval. It shows that the segment has the largest traffic demand. Then the averaged speed was analyzed; the total speed of the whole simulation time was $2.7669 e+003$. The averaged vehicle speed was 9.6072 and the maximum average was 16.6600. It appeared on the thirty-fourth segment in the seventh interval. It shows that the segment of the road was relatively smooth, and the vehicles have a higher speed.

When the proposed traffic organization optimization measures were implemented, the mentioned indexes were analyzed in the same way. The total density was 14.9110 in the whole simulation time. The averaged density is 0.0518 . The maximum averaged density is 0.2220 which was in the sixth interval on the fifth segment. They all have a little decline. It means that the proposed traffic organization method can balance the density on the network. The network density has been controlled; the congestion on the road has been greatly reduced. The total flow was 59982 . The averaged flow was 208.2708. The maximum averaged flow was 479, which appeared on the tenth segment of the sixth interval. It means that the capacity of the network has been greatly improved. The proposed traffic organization method was effective obviously. Moreover the averaged density and total

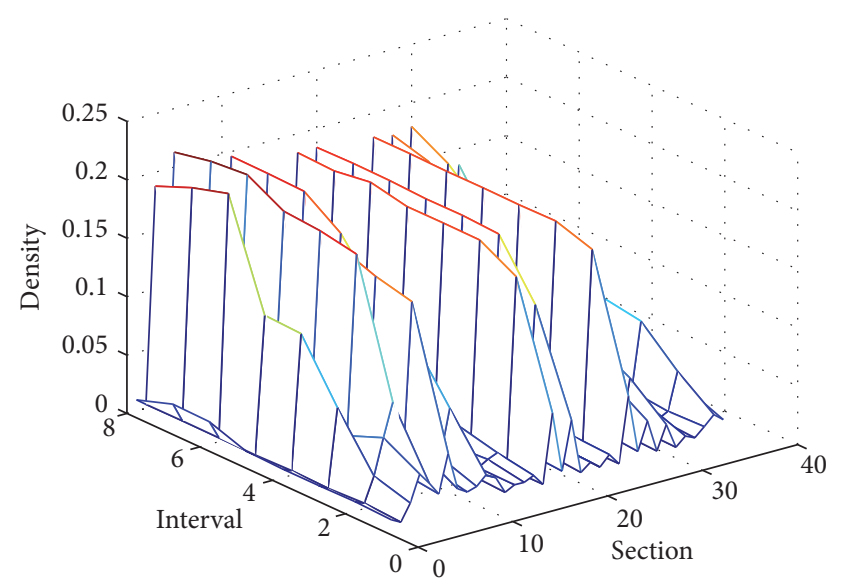

Figure 9: Averaged density after optimization.

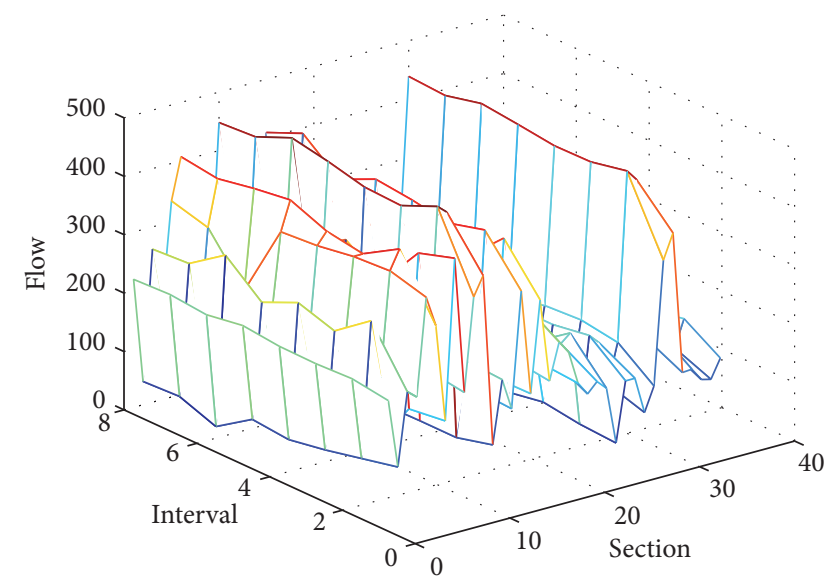

Figure 10: Averaged flow after optimization.

density were 7.8074 and $2.2485 e+003$, respectively. The maximum speed is 16.5300 appearing in the 25 th, 7 th, and 6 th intervals on 1st, 25th, and 28th segments. This means that the speed was smoothed on some segments which balanced the traffic flow. It was just the purpose of the traffic organization method.

The averaged density, speed, and flow on each segment of every interval were drawn out in Figures 6-11 before and after the traffic organization optimization measures were implemented.

\section{Conclusions}

In this paper, we overviewed the dynamic traffic simulation software DynaCHINA firstly which is one and only one self-developed software system in China by ours team. The improved traffic simulation model and algorithm were described in detail. The mesoscopic traffic simulation process was designed for suitable dynamic traffic analysis. An optimization method of regional traffic organization was proposed based on dynamic traffic analysis. The method was divided into two steps. First, the researched area was carefully channelized in the intersection level. Then, the traffic signal 


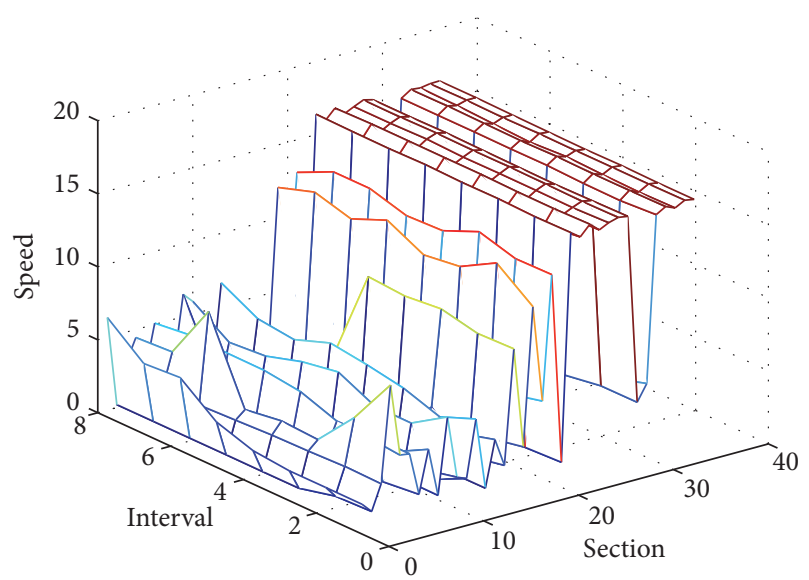

FIGURE 11: Averaged speed after optimization.

timings were optimized. The signal optimal control model was designed accordingly. Finally, the Daizong street of Tai'an city in Shandong province was used as a case study. The averaged density, averaged flow, and averaged speed on segments were selected as analytical index. The simulation results show that the proposed model and method were effective and feasible. The proposed method has been implemented to this area and has a good effect. The followed research will be the collaborative research on the road channelization and the traffic information optimization and the study of the realtime traffic simulation system. The optimization method will be extended to the traffic guidance information.

\section{Conflicts of Interest}

The authors declare that there are no conflicts of interest regarding the publication of this paper.

\section{Acknowledgments}

This work is supported by the National Natural Science Foundation of China (Grant nos. 71471104, 71371026, 71571109, and 71471167); the University Science and Technology Program Funding Projects of Shandong Province (Grant no. J14LI02); the Project of Public Security Department of Shandong Province (Grant no. GATHT2015-236); the Major Social and Livelihood Special Project of Jinan (Grant no. 20150905).

\section{References}

[1] Z. Zhai, Road Traffic Organization Optimization, China Communication Press, Beijing, China, 2004.

[2] F. Shi, E.-H. Huang, Q. Chen, and Y.-Z. Wang, "Optimization of one-way traffic organization for urban micro-circulation transportation network," Journal of Transportation Systems Engineering and Information Technology, vol. 9, no. 4, pp. 3035, 2009.

[3] D.-F. Long, F. Shi, and Y.-Z. Wang, "One-way traffic organization based on traffic load and road equity," Journal of Transportation Systems Engineering and Information Technology, vol. 10, no. 6, pp. 109-114, 2010.
[4] A. Dussutour, V. Fourcassié, D. Heibing, and J.-L. Deneubourg, "Optimal traffic organization in ants under crowded conditions," Nature, vol. 428, no. 6978, pp. 70-73, 2004.

[5] B. Jia, The study on traffic organization and optimization of urban arterial road [M.S. thesis], Chang'an University, Changan, China, 2010.

[6] H. Xu, Z. Tan, K. Long, and Z. Zhong, "Operational optimization method and simulation of bottleneck section based on urban arterial road," Computer and Communications, supplement 1, pp. 4-8, 2009.

[7] G.-Z. Yao, K.-Q. Wu, and H.-J. Wu, "Empirical analysis of the traffic Micro-circulation organization in heavy traffic networks," Journal of Transportation Systems Engineering and Information Technology, vol. 11, no. 1, pp. 160-166, 2011.

[8] Y.-Z. Yang, B.-H. Mao, X.-J. Zhang, R. Huang, and X.-J. Feng, "Study on traffic micro-circulation organization in heavy traffic networks based on route guidance," Journal of System Simulation, vol. 22, no. 7, pp. 1580-1584, 2010.

[9] B. H. Mao, Z. Z. Sun, S. P. Jia et al., Regional Traffic Organizational Optimization with Practice, China Communications Press, Beijing, China, 2007.

[10] L. Sun and F. Ren, "Optimize of the transfer organization in urban transportation terminal," Road Traffic and Safety, vol. 7, no. 3, pp. 18-21, 2007 (Chinese).

[11] L.-S. Sun, L.-Y. Yao, B.-H. Liu, Y.-Y. Wu, and J. Rong, “Traffic optimization of transportation terminal based on dynamic simulation technology," Journal of Beijing University of Technology, vol. 38, no. 4, pp. 570-574, 2012.

[12] W. Zhengyan, Study on the methods of traffic organization optimization for emergency evacuation [Ph.D. thesis], University of Jilin, 2011.

[13] Y. Lin and Y. Huang, "GLS model based dynamic OD origindestination matrix estimation for traffic systems," System Engineering-Theory \& Practice, vol. 1, pp. 136-144, 2004.

[14] K. Ashok and M. Ben-Akiva, "Dynamic origin-destination matrix estimation and prediction for real-time traffic management systems," in Proceedings of the 12th International Symposium on Transportation and Traffic Theory, pp. 465-484, Elsevier Science, Amsterdam, The Netherlands, 1993.

[15] Y. Lin, S.-B. Li, and Z. Jiang, "Parameter estimation for vehicle departure time distribution in process of dynamic OD flow loading," Journal of Beijing Institute of Technology (English Edition), vol. 21, no. 2, pp. 252-256, 2012.

[16] S. Li, Research on the run State evaluation of urban transportation system and control strategies [Ph.D. thesis], Beijing Jiaotong University, Beijing, China, 2012. 


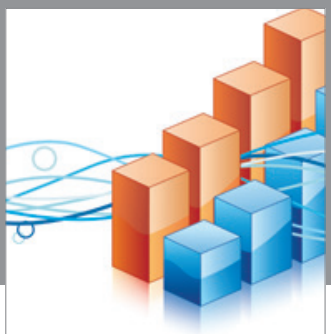

Advances in

Operations Research

vatem alat4

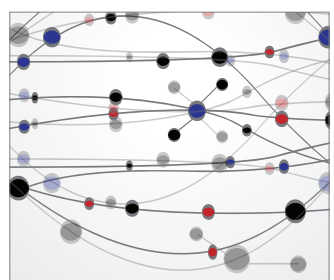

\section{The Scientific} World Journal
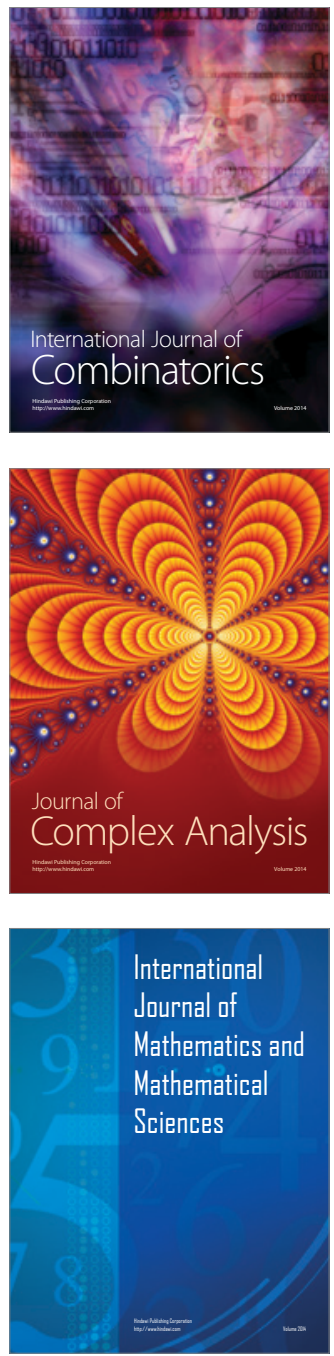
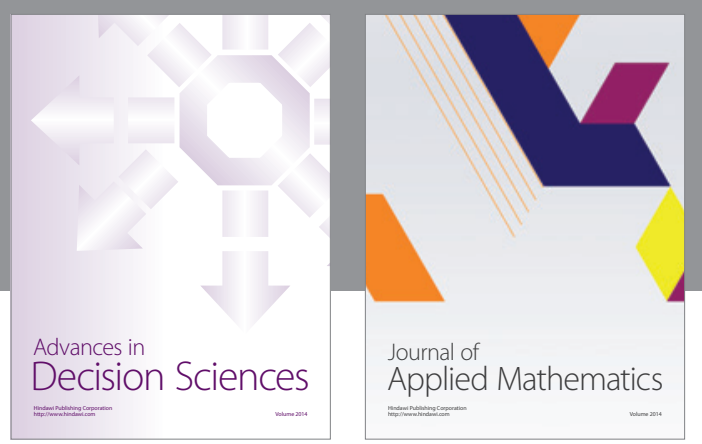

Algebra

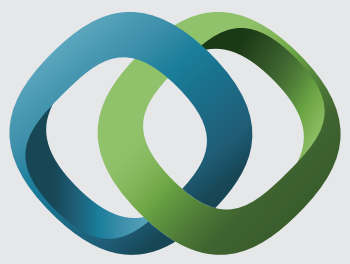

\section{Hindawi}

Submit your manuscripts at

https://www.hindawi.com
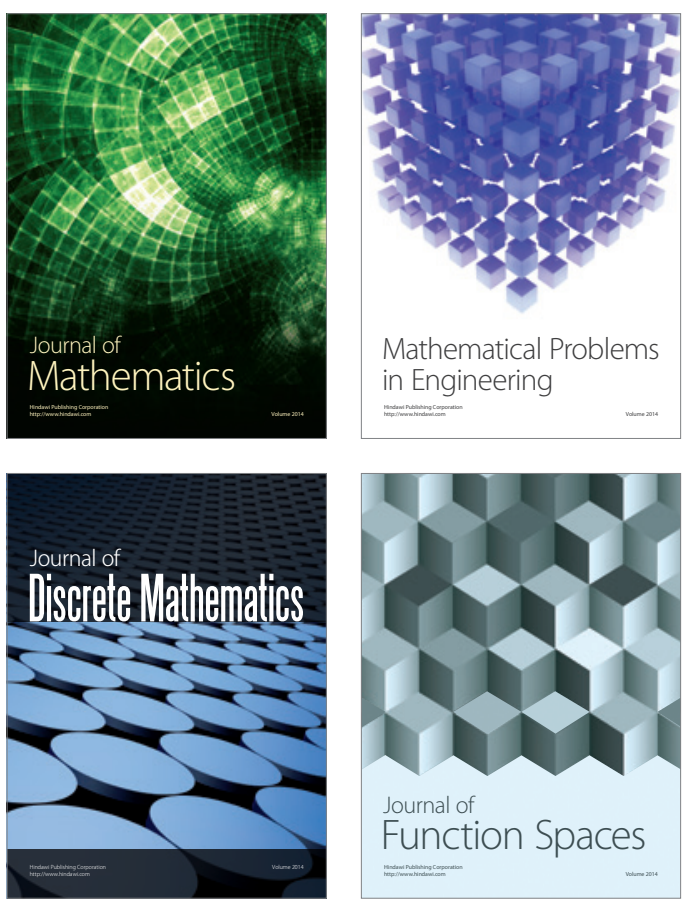

Mathematical Problems in Engineering
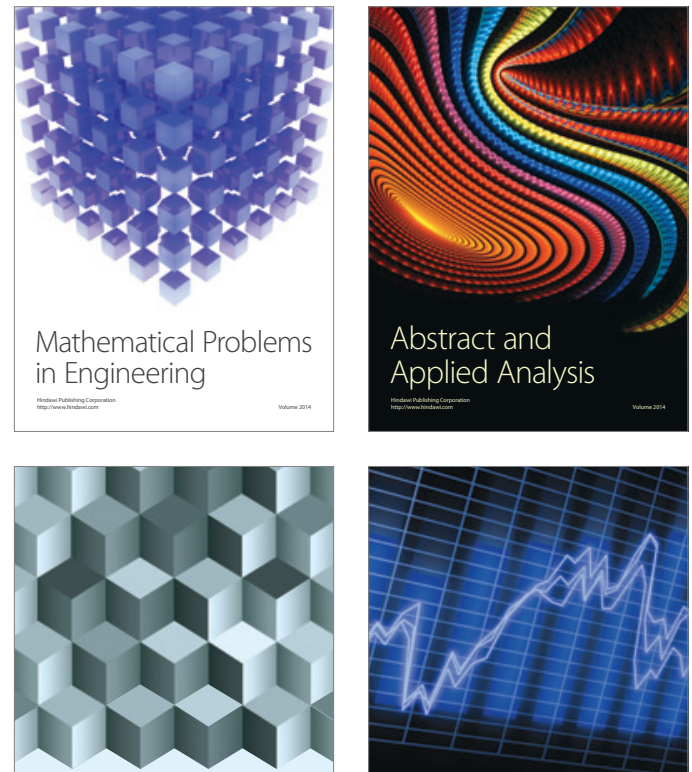

Journal of

Function Spaces

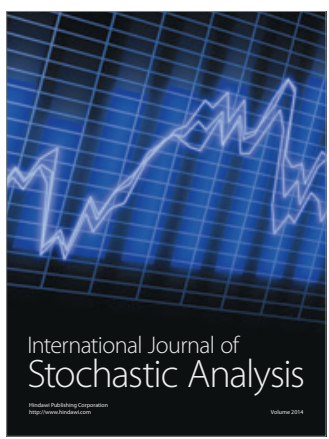

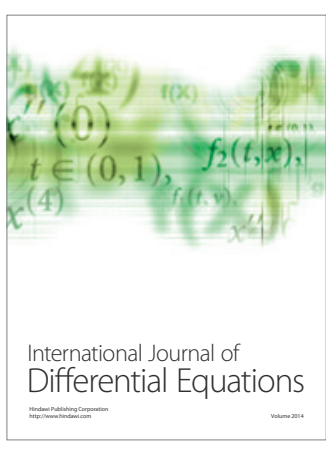
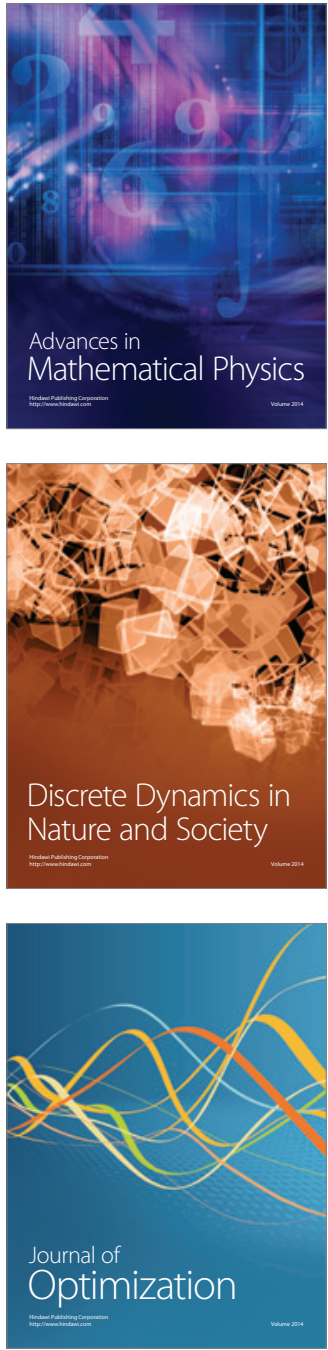